\title{
A Cable-net Form-finding Process for an Up-down Asymmetric Rim Truss
}

\author{
Yankang Ding ${ }^{1,2,3}$, Zihan Sun ${ }^{1,2,3}$, Dongwu Yang ${ }^{1,2,3}$, Yiqun Zhang ${ }^{1,2,3}$, and $\mathrm{Na} \mathrm{Li}^{1,2,3}$ \\ ${ }^{1}$ Key Laboratory of Electronic Equipment Structure Design, Ministry of Education, Xi'an, 710071, China \\ ${ }^{2}$ School of Electromechanical Engineering, Xidian University, Xi'an, 710071, China \\ ${ }^{3}$ Collaborative Innovation Center of Information Sensing and Understanding at Xidian University, Xi'an, 710071, China
}

\begin{abstract}
Traditional AstroMesh cable-net reflectors were deployed via a parallelogram mechanism, which provides symmetric supporting points for the front and rear nets and determines the height of stowed rim truss. In order to further reduce the height of stowed rim truss, a new up-down asymmetric rim truss was proposed without matched cable-net form-finding approach. This paper aims at designing a matched cable-net form-finding process for the new structure.
\end{abstract}

\section{Introduction}

With the rapid development of aerospace technology, high performance space antennas are in great demand for future space applications. Deployable cable-net reflector antennas with the advantages of low mass, thermal stability, and large scale have become more sophisticated over the past several decades and evolved many kinds of applications. As illustrated in Fig. 1, an AstroMesh [1] cable-net reflector consists of a deployable rim truss, two curved nets (i.e., a front net and a rear net) placed back-to-back, many adjustable ties for maintaining higher accuracy of the reflector, and an RF reflective mesh attached to the convex side of the front net.

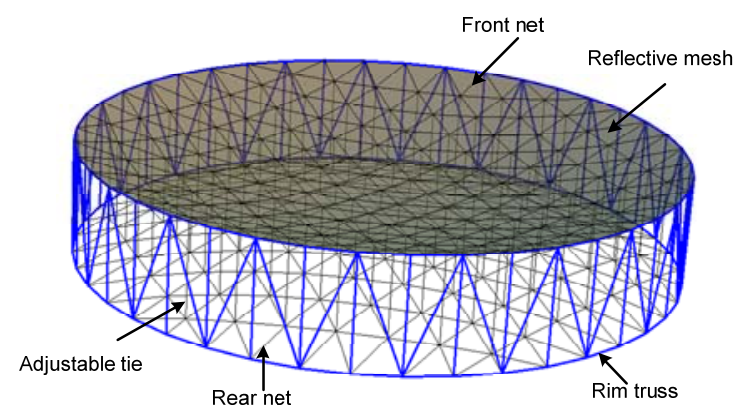

Fig. 1. Traditional AstroMesh reflector.

There are two well-accepted evaluation criteria in designing the cable-net of a mesh reflector (i.e. the surface accuracy and tension uniformity). Some scholars [2-4] proposed valid form-finding methods to meet the two criteria but only considering symmetric rim truss.

However, the height of stowed antenna packages will exceed the constraints of the Shuttle bay for an AstroMesh cable-net reflector antenna with a very large diameter. In an effort to solve this problem, Cao [5] proposed a kind of space deployable structure which can decrease the aspect ratio of height to diameter of stowed rim truss dramatically.

Compared with the traditional AstroMesh reflector, Cao's scheme, as depicted in Fig. 2, provides asymmetric supporting points on rim truss for the front and rear nets, which influences the topology of surface configuration and gives different boundary conditions for the front and rear nets. But there are no open literature describing the form-finding design process for an asymmetric rim truss at present. This paper is devoted to designing a cable-net form-finding process for the Cao's structure scheme, which may have a relatively small deformation once deployed.

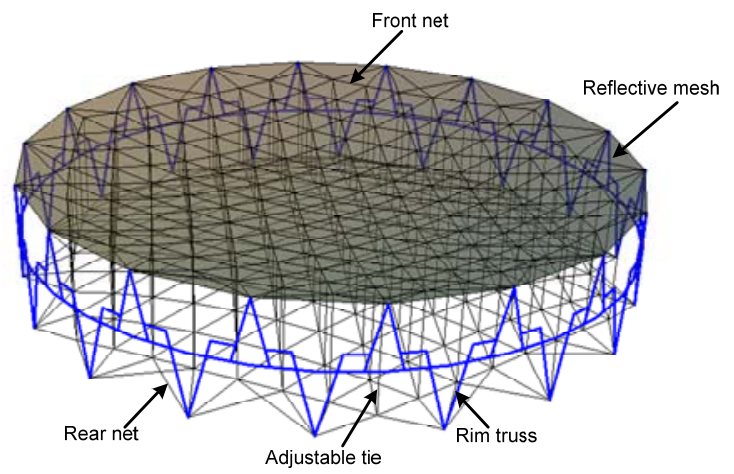

Fig. 2. Cao's structure scheme.

\section{Cable-net form-finding process}

\subsection{Equilibrium equations of the internal nodes}

For the cable-net reflector antennas, the front and rear nets are placed back-to-back, and they are both tensioned by adjustable ties. Every internal node $i^{\mathrm{f}}$ of the front net is connected by its corresponding node $i^{\mathrm{r}}$ on the rear net 
and a certain adjustable ties. A typical unit of the cable net is shown in Fig. 3.

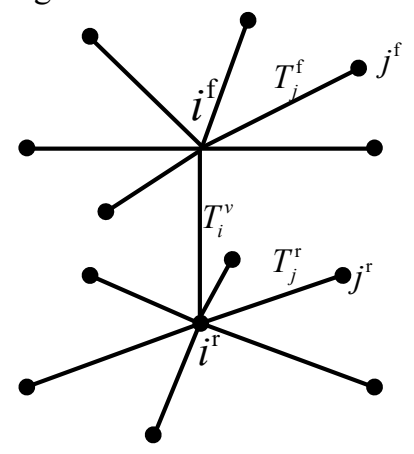

Fig. 3. A typical unit of the cable net.

There is no external force applied on the internal nodes whose equilibrium equations along $X$ direction of a coordinate system $(X, Y, Z)$ could be written as

$$
\begin{aligned}
& \sum_{j=1}^{c_{i}} T_{j}^{\mathrm{f}}\left(X_{j}^{\mathrm{f}}-X_{i}^{\mathrm{f}}\right) / L_{j}^{\mathrm{f}}+T_{i}^{\mathrm{v}}\left(X_{i}^{\mathrm{r}}-X_{i}^{\mathrm{f}}\right) / L_{i}^{\mathrm{v}}=0 \\
& \sum_{j=1}^{c_{i}} T_{j}^{\mathrm{r}}\left(X_{j}^{\mathrm{r}}-X_{i}^{\mathrm{r}}\right) / L_{j}^{\mathrm{r}}+T_{i}^{\mathrm{v}}\left(X_{i}^{\mathrm{f}}-X_{i}^{\mathrm{r}}\right) / L_{i}^{\mathrm{v}}=0
\end{aligned}
$$

where $X_{i}^{\mathrm{f}}$ and $X_{i}^{\mathrm{r}}$ represent the $X$ coordinate of the nodes $i^{\mathrm{f}}$ and $i^{\mathrm{r}}$. The same equations could also be written in the $Y$ and $Z$ directions.

\subsection{Topology design}

For Cao's scheme, the front and rear nets of cable-net antennas are always asymmetric, which was taken into consideration on the phrase of topology design.

To determine the cable-net topology, four steps have to be implemented. The procedure is stated in detail below. Firstly, a 6 by $n$ initial configuration, as shown in Fig. $4 \mathrm{a}$, should be constructed. For a certain requirement on the reflector surface accuracy, the $n$ could be determined by reference [6,7]. And the boundary nodes are determined by the $n$ and the diameter of reflector antenna. All nodes of the initial configuration should locate on the desired paraboloidal surface. Secondly, all nodes on a uniform tension configuration as shown in Fig. $4 \mathrm{~b}$ could be calculated by using the equal-force form-finding method [8]. Thirdly, the final configuration of the front net as shown in Fig. 4c could be obtained by reconnecting boundary cables while reserving the inner cables of the uniform tension configuration. Finally, the final configuration of the rear net as shown in Fig. 4d could also be acquired via the same method as the front net. It is worth noting that the four steps could promise the symmetry of inner cables of both nets.

\subsection{Form-finding process with rigid rim truss}

In this section, the rim truss was treated as rigid. Based on the results of topology design, boundary conditions of both nets could be determined while the equal force state of the inner nets was reserved. The procedure of form-finding could be depicted as the following.
Step 1. Based on the final configuration of both nets, an initial force distribution could be obtained by using the least-norm method $[9,10]$ respectively. It should be noted that the force equilibrium of $X$ and $Y$ directions could not promise $Z$ direction because of the different topology of the front and rear nets.

Step 2. Calculate the forces of adjustable ties $T_{i}^{\mathrm{v}}$. Based on the current configuration of the front net and the current directions of the adjustable ties, $T_{i}^{\mathrm{v}}$ could be acquired by projecting every cable force of the front net connected with node $i^{\mathrm{f}}$ on the current directions of the adjustable ties and summing those component forces.

Step 3. Haven known all cable forces and all cable length, equilibrium equations could be linearized and solved by introducing force density coefficients. Those force density coefficients are defined by

$$
q_{j}^{\mathrm{f}}=T_{j}^{\mathrm{f}} / L_{j}^{\mathrm{f}}, q_{j}^{\mathrm{r}}=T_{j}^{\mathrm{r}} / L_{j}^{\mathrm{r}}, q_{i}^{\mathrm{v}}=T_{i}^{\mathrm{v}} / L_{i}^{\mathrm{v}}
$$

Eqs. (1) and (2) are linearized as

$$
\begin{aligned}
& \sum_{j=1}^{c_{i}} q_{j}^{\mathrm{f}}\left(X_{j}^{\mathrm{f}}-X_{i}^{\mathrm{f}}\right)+q_{i}^{\mathrm{v}}\left(X_{i}^{\mathrm{r}}-X_{i}^{\mathrm{f}}\right)=0 \\
& \sum_{j=1}^{c_{i}} q_{j}^{\mathrm{r}}\left(X_{j}^{\mathrm{r}}-X_{i}^{\mathrm{r}}\right)+q_{i}^{\mathrm{v}}\left(X_{i}^{\mathrm{f}}-X_{i}^{\mathrm{r}}\right)=0
\end{aligned}
$$

New space coordinates of all internal nodes could be obtained by solving those equilibrium equations of $X, Y$, and $Z$ directions.

Step 4. To promise the accuracy of the mesh reflector, the internal nodes of the front net should be positioned on the desired paraboloidal surface, and then the $Z$ coordinates of those front-net nodes are recalculated by the following Eq. (6).

$$
Z_{i}^{\mathrm{f}}=\left(X_{i}^{\mathrm{f} 2}+Y_{i}^{\mathrm{f} 2}\right) /(4 F) \quad\left(i=1,2, \cdots, n_{i n}\right)
$$

Step 5. Looping through steps 2 to 4 until the norm of its coordinate variations is inferior to the tolerance allowed.
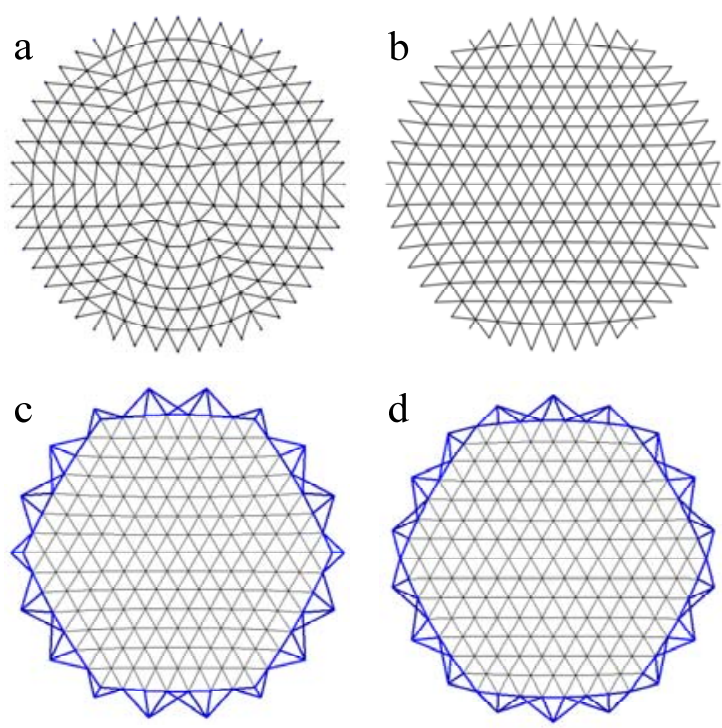

Fig. 4. A topology design procedure: (a) initial configuration; (b) equal-force configuration; (c) final configuration of the front net; (d) final configuration of the rear net. 


\subsection{Form-finding process with elastic rim truss}

With the increasement of the antenna aperture, the assumption that the rim truss is rigid is not reasonable. The elastic deformation of the rim truss have to be considered in our form-finding method with the help of the commercial software $A N S Y S$. In the finite element model of ANSYS, beam188 elements are used to model rim truss and link10 elements are used to model the cable net with tension forces.

The design approach could be realized by an iterative procedure as the following.

Step 1. Construct an initial finite element model. After topology design, the geometrical configuration will not change at all. Based on the final configuration, the cable forces of the front and rear nets could be obtained by using the from-finding process with rigid rim truss. Then, an initial finite element model of the whole reflector antenna is constructed. In this model, the preloads $T$ of the cables is applied by an initial strain

$$
\varepsilon=T /(E A)
$$

where $E$ and $A$ represent the elastic module and the cross section area of the cable element respectively.

Step 2. Obtain a new boundary condition. A static analysis of the current model should be performed to find the deformation results which include the positions of the connection nodes of the cable net and the nodes on the rim truss. A new boundary condition is determined by the space position of the nodes on the rim truss in the deformed shape.

Step 3. Find a best-fit paraboloid. Suppose that a best-fit paraboloid is acquired by rotating $\theta_{x}, \theta_{y}, \theta_{z}$ around the $X, Y$, and $Z$ axis respectively and shifting $u, v, w$ along the $X, Y$, and $Z$ axis respectively. A kind of optimized model was proposed at the aim of minimizing root-mean-square error. The optimized model can be expressed as follows

$$
\begin{array}{ll}
\text { Find } & \boldsymbol{s}=\left[\theta_{x} \theta_{y} \theta_{z} u v w\right]^{\mathrm{T}} \\
\text { Min } & \mathrm{rms}=\sqrt{\sum_{i=1}^{m}\left({ }^{n e w} Z_{i}-\frac{{ }^{n e w} X_{i}^{2}+{ }^{n e w} Y_{i}^{2}}{4 F}\right)^{2} / m} \\
\text { s.t. } & |u|,|v|,|w| \leq D \\
& \left|\theta_{x}\right|,\left|\theta_{y}\right|,\left|\theta_{z}\right| \leq \theta_{\mathrm{lim}}
\end{array}
$$

where $m$ is the number of internal nodes of the front net; $D$ and $F$ are antenna aperture diameter and focal length, respectively; $\theta_{\lim }$ is the limit of rotation angle allowed.

By solving this optimized model, the parameters which determine a best-fit paraboloid are obtained and the positions of all internal nodes of the both nets should be updated by the Eq.(9).

$$
\left[{ }^{\text {new }} X,{ }^{\text {new }} Y,{ }^{\text {new }} Z\right]^{\mathrm{T}}=\left([X, Y, Z]^{\mathrm{T}}-[u, v, w]^{\mathrm{T}}\right) \cdot R_{z} \cdot R_{y} \cdot R_{x}
$$

where

$$
R_{z}=\left[\begin{array}{ccc}
\cos \theta_{z} & -\sin \theta_{z} & 0 \\
\sin \theta_{z} & \cos \theta_{z} & 0 \\
0 & 0 & 1
\end{array}\right], \quad R_{y}=\left[\begin{array}{ccc}
\cos \theta_{y} & 0 & \sin \theta_{y} \\
0 & 1 & 0 \\
-\sin \theta_{y} & 0 & \cos \theta_{y}
\end{array}\right],
$$

$$
R_{x}=\left[\begin{array}{ccc}
1 & 0 & 0 \\
0 & \cos \theta_{x} & -\sin \theta_{x} \\
0 & \sin \theta_{x} & \cos \theta_{x}
\end{array}\right] .
$$

Step 4. Form finding with new boundary conditions. Based on the updated nodes and new boundary nodes, one more cable net form-finding is performed by using the form-finding process with rigid rim truss again. The form-finding results are used for updating the finite element model, and then the cable force and the length of cables could be obtained after another static analysis. Denote the current strain of a cable by $\varepsilon_{1}$, the initial strain for this cable in the initial finite element model is calculated by

$$
\varepsilon_{0}=\varepsilon_{1}+\Delta L / L_{0}
$$

where $\Delta L$ is the variable quantity of the cable length; $L_{0}$ is the initial cable length in the initial finite model.

Step 5. Looping through Steps 2 to 4 until the value of $\varepsilon_{0}$ reached to the initial strain $\varepsilon$.

\section{Numerical examples}

In order to illustrate the proposed form-finding process, a numerical example is discussed here for a cable-net reflector antenna with $D=5 \mathrm{~m}$ and $F=4 \mathrm{~m}$. The material and geometrical parameters of the elements in the finite element model was tabulated in Table 1. The tension distribution of the front and rear nets are symmetrical. So one-sixth of the results are shown in Fig. 5 and Fig. 6.

Table 1. Material and geometrical parameters of the elements.

\begin{tabular}{|c|c|c|c|}
\hline & $\begin{array}{l}\text { Maximum } \\
\text { force }(\mathrm{N})\end{array}$ & $\begin{array}{l}\text { Minimum } \\
\text { force }(\mathrm{N})\end{array}$ & Max/Min \\
\hline Front cables & 45 & 20 & 2.25 \\
\hline Rear cables & 62 & 20 & 3.10 \\
\hline $\begin{array}{l}\text { Adjustable } \\
\text { ties }\end{array}$ & 4.59 & 1.98 & 2.32 \\
\hline \multicolumn{2}{|c|}{ Maximum deformation } & \multicolumn{2}{|c|}{$10.98 \mathrm{~mm}$} \\
\hline \multicolumn{2}{|c|}{$\begin{array}{c}\text { Faceting error of effective } \\
\text { zone }\end{array}$} & \multicolumn{2}{|c|}{$0.49 \mathrm{~mm}$} \\
\hline \multicolumn{2}{|c|}{ Faceting error of whole zone } & \multicolumn{2}{|c|}{$0.74 \mathrm{~mm}$} \\
\hline
\end{tabular}

\begin{tabular}{|c|c|c|}
\hline Element type & Parameter & value \\
\hline \multirow{3}{*}{ Link10 } & Cross section area & $0.785 \mathrm{~mm}^{2}$ \\
\cline { 2 - 3 } & Elastic modulus & $20 \mathrm{Gpa}$ \\
\cline { 2 - 3 } & Poisson ratio & 0.3 \\
\hline \multirow{4}{*}{ Beam188 } & Inner diameter & $16 \mathrm{~mm}$ \\
\cline { 2 - 3 } & Outer diameter & $18 \mathrm{~mm}$ \\
\cline { 2 - 3 } & Elastic modulus & $210 \mathrm{Gpa}$ \\
\cline { 2 - 3 } & Poisson ratio & 0.3 \\
\hline
\end{tabular}

The primary information of the cable-net reflector is concluded in Table 2 and Fig. 7 gives the deformation graph, where the displacement was scaled by a factor of 10. The maximum deformation is $10.98 \mathrm{~mm}$ with an accuracy of $0.49 \mathrm{~mm}$, which indicates the feasibility of this form-finding process.

Table 2. Key information of the cable-net reflector. 


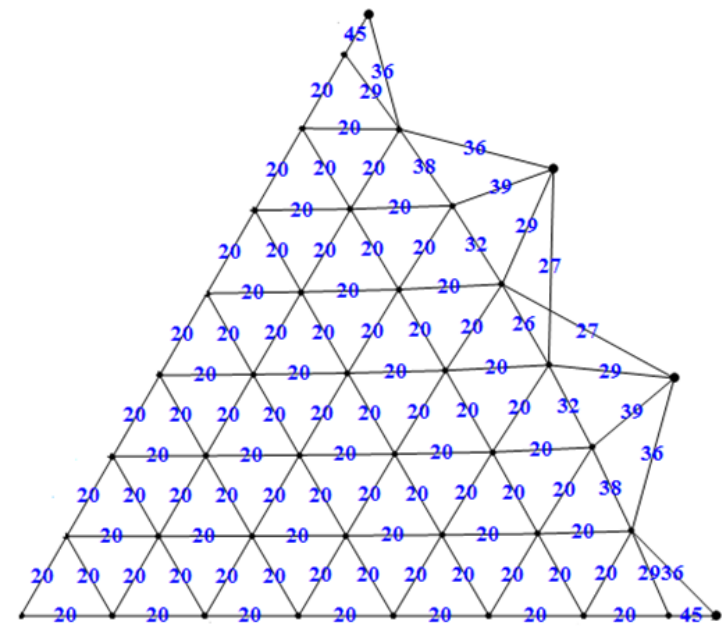

Fig. 5. The tension distribution of the front net.

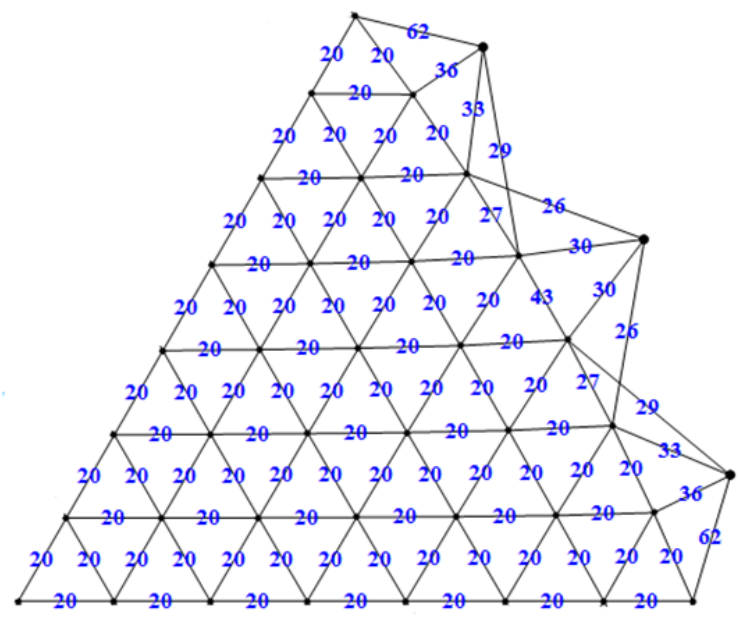

Fig. 6. The tension distribution of the rear net.

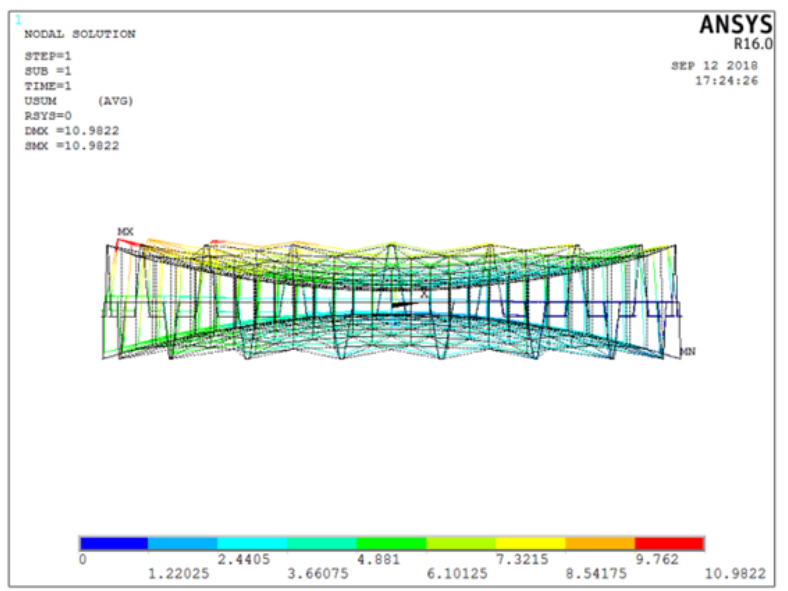

Fig. 7. Deformation graph of the whole reflector.

\section{Conclusion}

A cable-net form-finding procedure is proposed to match the new up-down asymmetric space structure. A numerical example is carried out and the results demonstrate the correctness and effectiveness of the proposed form-finding procedure. As two well-admitted evaluation criteria, the surface accuracy and the tension uniformity shown in the numerical example are also satisfied.

This work is supported by the National Natural Science Foundation of China with No.51775404, Nature Science Basic Research Plan in Shaanxi Province with No. 2016JQ5072 \& No. 2016JQ5006, Fundamental Research Funds for the Central Universities with No. JB180410, and the Shanghai Aerospace Science and Technology Innovation Fund.

\section{References}

1. M. Thomson, The AstroMesh deployable reflector [C]. Antennas and propagation Society, IEEE Int. Symp. 3, 1516-1519 (1999).

2. T. Li, J. Jiang, H. Deng, Z. Lin, Z. Wang, Form-finding methods for deployable mesh reflector antennas[J], Chin. J. Aeronaut. 26, 1276-1282 (2013).

3. X. Ma, Y. Song, Z. Li, T. Li, Z. Wang, H. Deng, Mesh reflector antennas: form-finding analysis review, Proceedings of $54^{\text {th }}$ AIAA Structures, Structural Dynamics, and Materials Conference, AIAA, Boston, Massachusetts, (2013).

4. W. Liu, D. Li, X. Yu, J. Jiang, Exact mesh shape design of large cable-network antenna reflectors with flexible ring truss supports[J], Acta Mechanics Sinica 30, 198-205 (2014).

5. P. Cao, H. Bao, B.Y. Duan, A deployable structure of space planar membrane antenna, $\mathrm{CN}$, 201710607804.1[P].2017-12-08.(In Chinese)

6. P. K. Agrawal, M.S. Anderson, M.F. Card, Preliminary design of large reflectors with flat facets[J], IEEE Trans. Antenna. Propag. 29 (4), 688694 (1981).

7. D.W. Yang, S.X. Zhang, T.J. Li, Preliminary design of paraboloidal reflectors with flat facets[J], Acta Astronaut. 89, 14-20 (2013).

8. S. Morterolle, B. Maurin, J. Quirant, C. Dupuy, Numerical form-finding of geo-tensoid tension truss for mesh reflector[J], Acta Astronaut. 76, 154-163 (2012).

9. D. Yang, G. Yang, Y. Zhang, J. Du, Least-squares minimization of boundary cable tension ratios for mesh reflectors [J], AIAA J. 56 (2), 883-888 (2018).

10. D. Yang, Y. Qiu, H. Bao, Least-norm Method for Pretension Optimization of Mesh Reflector[J], Journal of Mechanical Engineering, 48(21), 22-27 (2012). (In Chinese). 